\title{
A!
}

This is an electronic reprint of the original article.

This reprint may differ from the original in pagination and typographic detail.

Ponnada, Tushara; Kazemi, Parham; Al-Tous, Hanan; Liang, Ying-Chang; Tirkkonen, Olav

\section{Best Beam Prediction in Non-Standalone mm Wave Systems}

Published in:

2021 Joint European Conference on Networks and Communications \& 6G Summit (EuCNC/6G Summit)

DOI:

10.1109/EuCNC/6GSummit51104.2021.9482504

Published: $28 / 07 / 2021$

Document Version

Peer reviewed version

Please cite the original version:

Ponnada, T., Kazemi, P., Al-Tous, H., Liang, Y-C., \& Tirkkonen, O. (2021). Best Beam Prediction in Non-

Standalone mm Wave Systems. In 2021 Joint European Conference on Networks and Communications \& 6G

Summit (EuCNC/6G Summit) (pp. 532-537). [9482504] (European conference on networks and

communications). IEEE. https://doi.org/10.1109/EuCNC/6GSummit51104.2021.9482504

This material is protected by copyright and other intellectual property rights, and duplication or sale of all or part of any of the repository collections is not permitted, except that material may be duplicated by you for your research use or educational purposes in electronic or print form. You must obtain permission for any other use. Electronic or print copies may not be offered, whether for sale or otherwise to anyone who is not an authorised user. 


\title{
Best Beam Prediction in Non-Standalone mmWave Systems
}

\author{
Tushara Ponnada*, Parham Kazemi*, Hanan Al-Tous*, Ying-Chang Liang ${ }^{\dagger}$, Olav Tirkkonen* \\ * Department of Communications and Networking, Aalto University, Espoo, Finland \\ $\dagger$ University of Electronic Science and Technology of China, Chengdu, P. R. China \\ Email:\{tushara.ponnada, parham.kazemi, hanan.al-tous, olav.tirkkonen\}@aalto.fi, liangyc@ieee.org
}

\begin{abstract}
We consider a machine learning approach to perform best beam prediction in Non-Standalone Millimeter Wave (mmWave) Systems utilizing Channel Charting (CC). The approach reduces communication overheads and delays associated with initial access and beam tracking in 5G New Radio (NR) systems. The network has a mmWave and a sub-6 GHz component. We devise a Base Station (BS) centric approach for best mmWave beam prediction, based on Channel State Information (CSI) measured at the sub-6 GHz BS, with no need to exchange information with UEs. In a training phase, we collect CSI at the sub-6 GHz BS from sample UEs, and construct a dimensional reduction of the sample CSI, called a $\mathrm{CC}$. We annotate the $\mathrm{CC}$ with best beam information measured at a mmWave BS for the sample UEs, assuming autonomous beamformer at the UE side. A beam predictor is trained based on this information, connecting any sub-6 GHz CSI with a predicted best mmWave beam. To evaluate the efficiency of the proposed framework, we perform simulations for a street segment with synthetic spatially consistent CSI. With a neural network predictor, we obtain 91\% accuracy for predicting best beam and $99 \%$ accuracy for predicting one of two best beams. The accuracy of $\mathrm{CC}$ based beam prediction is indistinguishable from true location based beam prediction.
\end{abstract}

Index Terms-Non-Standalone systems, beam prediction, channel charting, network centric approach, radio resource management

\section{INTRODUCTION}

Millimeter Wave (mmWave) communication, offering wide bands, is a key enabling technology of Fifth Generation (5G) and Beyond 5G (B5G) communication systems to handle the explosive demand for high data rates. Beamforming and Massive Multiple-Input-Multiple-Output (mMIMO) antenna arrays at the Base Stations (BSs) are expected to provide directionality and beamforming gain to combat the high propagation loss that characterizes mmWave communications. Because of the susceptibility of mmWave communications to blockage, precise beams alignment is necessary for maintaining quality of service [1].

To improve the robustness of mmWave communications, multi-connectivity approaches are adopted. In Non-Standalone systems (NSA), mmWave communication is used in combination with sub-6 $\mathrm{GHz}$ communication (e.g., based on Long Term Evolution (LTE)), which improves robustness of mmWave communications and boosts performance [2]. Sub$6 \mathrm{GHz}$ communication can be used for control channels for the mmWave system, or to provide coverage with limited data rate, if the mmWave channel is blocked. Such dual connectivity not only helps in improving performance, but it also aids in smooth transition and interconnectivity of upcoming B5G networks with legacy LTE networks.

Beam management procedures are designated by $3 \mathrm{GPP}$ to continually determine the optimal beams for communication in 5G New Radio (5GNR). Beam management includes learning the optimal beam for transmission in case of an idle User Equipment (UE) which has to establish a connection with the BS for the first time. This is called 'Initial Access' [3]. For connected UEs, mmWave channel quality may rapidly change. Accordingly, beam adaptation and handover procedures to maintain accurate beam alignment, known as 'Beam Tracking', are needed. In cellular mmWave systems, it is important to have efficient procedures for Initial Access and Beam Tracking to facilitate reliable communications. In 5GNR, the UE autonomously selects a beam direction to transmit to in uplink, and to receive from in downlink, so that UE beamforming is not under the control of the mmWave BS. This makes the problem of determining the best BS beam a non-trivial problem for mmWave 5GNR systems. Even with a fully digital antenna array, a BS cannot determine the best combination of BS and UE beams.

In 5GNR, the large number of beams makes conventional beam sweeping approaches for beam management challenging. Exploiting location side information has been shown to be beneficial in speeding up beam training [4]. Location assisted beam management combined with Machine Learning (ML) techniques is discussed in [5] for multi-cell and in [6] for single-cell scenario. Neighbor assisted beam search has been proposed in [7] for fast beam sweeping. First, the closest user's beam is explored and then gradually the search moves to other beams. Alternatively the line-of-sight-angles of a user and its nearest neighbor can be used. In these approaches, the geolocation of UEs is assumed to be known. In [8], deep learning models are used to predict optimal beams and blockage status of mmWave communications using the instantaneous sub- $6 \mathrm{GHz}$ raytraced channels in a dual band system.

Channel Charting is based on applying unsupervised ML techniques to learn the logical map of the radio environment. The Channel State Information (CSI) of sample UEs is dimensionally reduced to a low-dimensional radio-map of a cell [9], called a Channel Chart (CC), which can play a significant role in performing Radio Resource Management (RRM) functions.

In this paper, we explore the possibility of using $\mathrm{CCs}$ for best beam prediction of NSA mmWave systems. We consider 
a CC-based network-centric framework for best mmWave beam prediction for a UE, based on long term CSI measured by a possibly different $\mathrm{BS}$ at a sub- $6 \mathrm{GHz}$ carrier. The considered framework can be utilized both for Initial Access and for Beam-Tracking for mobile UEs without the use of their true physical locations. The UEs best mmWave beam will be predicted at a network level, thus avoiding the delay and communication overhead for determining the best BS beam at the UE's side and then updating the BS with the best beam information. We predict the best BS beam for a new UE location using a $\mathrm{CC}$ annotated with best beam information in a training phase, and a supervised ML prediction algorithm.

The rest of this paper is organized as follows. In Section II, the system model is presented. In Section II-B, CC basic concepts are introduced. In Section III-B, best beam prediction techniques are proposed. Simulation settings and numerical results are presented and discussed in Section IV. Lastly, conclusions are presented in Section V.

\section{System ModeL}

We consider a NSA mmWave communication system, where in the coverage area of a sub- $6 \mathrm{GHz}$ BS there may be many mmWave BSs. Without loss of generality, we concentrate on the problem of predicting the best beams of one mmWave BS.

\section{A. Channel Model}

For the sub- $6 \mathrm{GHz}$ communication, the BS has an array of $P$ antenna elements and the UEs have a single omnidirectional antenna. A set of $U$ UEs are present in the network. In the sub-6 GHz frequency, the mobile UEs perform pilot transmissions and the BS measures the channel $\mathbf{h}_{\mathbf{u}} \in \mathbb{C}^{P \times 1}$. The CSI covariance of UE $u$ at the sub- $6 \mathrm{GHz}$ BS is computed as:

$$
\mathbf{R}_{u}=\mathbb{E}\left\{\mathbf{h}_{u} \mathbf{h}_{u}^{H}\right\}
$$

where the expectation $E\{$.$\} is over a number of temporal$ samples of the fast fading process of the mobile user, within a short time window, and over frequency domain subcarriers, and $(.)^{H}$ denotes the Hermitian conjugate.

MmWave communication is based on Time-DivisionDuplexing (TDD), assuming channel reciprocity. The mmWave BS has an antenna array with $M$ elements while the UEs have $N$ antennas. Both the BS and UE are capable of beamforming and communicate through discrete sets of beams. We assume $B=M$ beams for the $\mathrm{BS}$ and $B_{u}=N$ beams for the UE. For simplicity, we assume that the BS has $M$ Radio Frequency (RF) chains, such that it can simultaneously transmit/receive from multiple beams. We consider wideband BS beams, i.e., the same beam is used for all subcarriers. To get an upper bound on performance, we assume that UEs are capable of digital beamforming, and can change the beam for each subcarrier. Both the BS and the UEs beamformers are based on Discrete Fourier Transform (DFT) codebooks with beams

$$
\mathbf{c}_{q}=\frac{1}{\sqrt{Q}}\left[1, e^{j 2 \pi \frac{q}{Q}}, \ldots, e^{j 2 \pi \frac{(Q-1) q}{Q}}\right]^{T}, q=0, \ldots, Q-1 .
$$

Here $Q=M$ for the BS codebook and $Q=N$ for the UE codebook.

The MIMO channel between the mmWave BS and UE $u$ on a subcarrier is $\mathbf{G}_{u} \in \mathbb{C}^{M \times N}$. The received signal at mmWave BS beam $\mathbf{w}_{m}$ from UE $u$ transmitting on beam $\mathbf{v}_{n}$ then is:

$$
y_{m, n}^{u}=\mathbf{w}_{m}^{H} \mathbf{G}_{u} \mathbf{v}_{n} x+z_{u}=g_{m, n}^{u} x+z_{u},
$$

where $g_{m, n}^{u}=\mathbf{w}_{m}^{H} \mathbf{G}_{u} \mathbf{v}_{n}$ is the effective channel of UE $u$ using beam $n$ received at BS beam $m, x$ is the transmitted symbol with $\mathbb{E}\left\{|x|^{2}\right\}=1$ and $z_{u}$ is noise.

Given a downlink transmission of the BS on beam $m$, the UE can determine its corresponding best beam as:

$$
\hat{n}(m)=\arg \max _{n}\left|g_{m, n}^{u}\right|^{2} .
$$

The BS cannot determine the UE beam from the uplink received signal at the BS. Due to autonomous UE beamforming, a set of downlink transmissions on all BS beams $\mathbf{w}_{m}$ is needed to find the best beam combination. For each $m$, the UE finds the best beam $\hat{n}(m)$ and the corresponding effective signal power. The best BS beam for the UE can then be found from

$$
m_{u}^{*}=\arg \max _{m} \mathbb{E}\left\{\left|g_{m, \hat{n}(m)}^{u}\right|^{2}\right\}
$$

where the expectation is over subcarriers and temporal fast fading samples. This beam index is then fed back by the UE to the $\mathrm{BS}$ in a beam management procedure. Note that the best BS beam is averaged over temporal samples within a short time window, while the UE selects its beam based on instantaneous channels.

The objective of this work is to eliminate the need for multiple transmissions and feedback for finding the best BS beam, directly predicting the best mmWave BS beam from sub-6 GHz CSI.

\section{B. Basics of Channel Charting}

Channel charting is the process of learning the radio maps which embody the network layout and UE neighborhood relations in terms of their physical location neighborhood relations [9]. The basic idea of Channel charting is to create a 2D/3D representation of the radio environment from the CSI at MIMO BSs so that the chart distances conform to the true physical distances between the UEs, i.e., UEs which are near in the physical domain are near in the chart and similarly for far UEs. In channel charting, CSI features $\boldsymbol{f}_{u}$ are first extracted for each UE $u$. Then, the set of features $\left\{\boldsymbol{f}_{u}\right\}_{u=1}^{U}$ is transformed to a lower dimension using Dimensionality Reduction (DR), typically based on pairwise feature distances between the UEs.

In this paper, we shall use the sub- $6 \mathrm{GHz}$ covariance matrices $\left\{\mathbf{R}_{u}\right\}_{u=1}^{U}$ of the UEs as features. To measure the feature dissimilarity, we use the Log-Euclidean distance. It provides a geodesic distance for positive semi-definite matrices [10], and has low computational complexity. The dissimilarity between the covariance features of UEs $u$ and $u^{\prime}$ is then:

$$
d_{u, u^{\prime}}=\left\|\log \mathbf{R}_{u}-\log \mathbf{R}_{u^{\prime}}\right\|_{\mathrm{F}},
$$



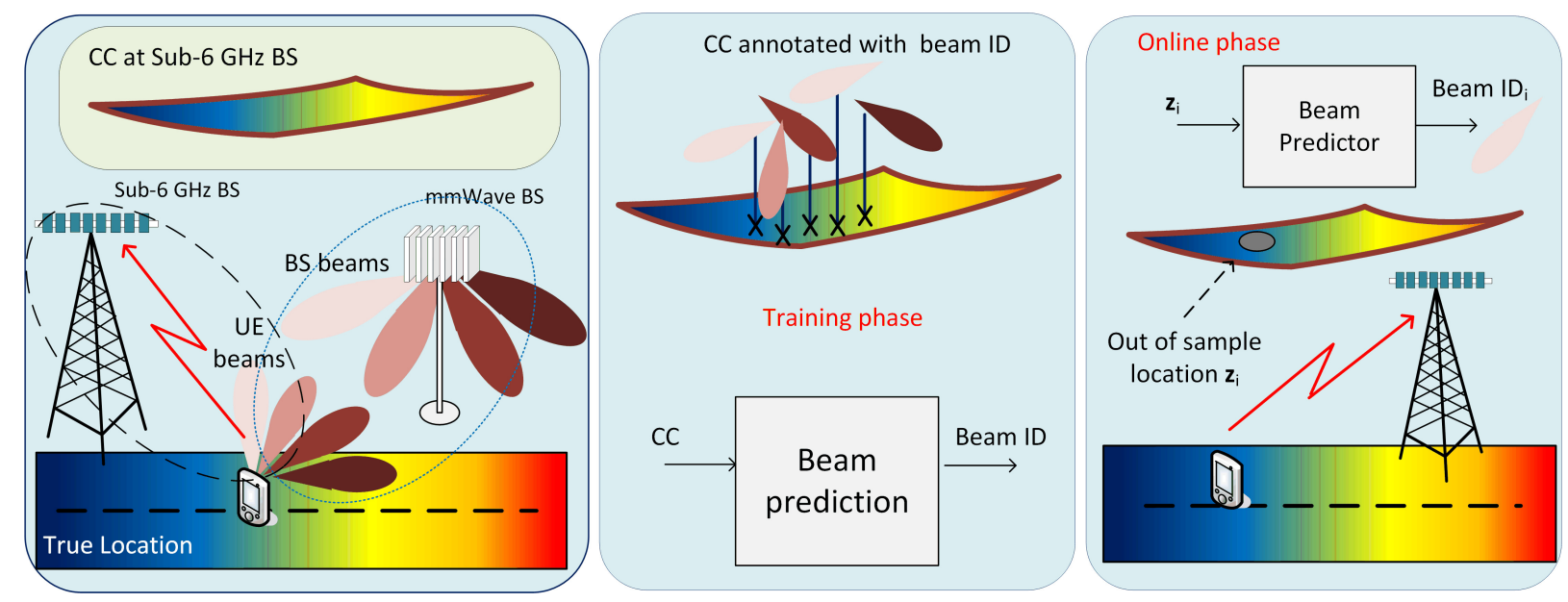

Fig. 1: Beam prediction based on channel charting in Non-Standalone systems. (Left); a street segment served by a multi antenna sub-6 GHz and mmWave BSs. (Middle); Training phase with CC construction and annotation. (Right); Online phase showing the best beam prediction for a new UE.

where the matrix logarithm is used. The dissimilarity matrix is fed to a DR algorithm to obtain a $2 \mathrm{D}$ representation of the CSI of the set of UEs, the CC.

For DR, we shall use ISOMAP, and $t$-Distributed Stochastic Neighbor Embedding ( $t$-SNE) [11]. The obtained charts are expected to preserve neighborhood relations of the physical locations of the UEs. To check the quality of the CCs, we employ three quality metrics; Trustworthiness (TW), Continuity (CT) and Kruskal Stress (KS), see [9], [12]. CT indicates how well the physical domain neighbors are preserved in the representation domain, while TW measures if the representation introduces neighbors that did not exist in the physical domain. KS measures the global similarity of the physical and charting locations of the UEs. The range of all three metrics is $\left[\begin{array}{ll}0 & 1\end{array}\right]$. For an ideal representation, fully reproducing the physical distances, CT and TW are 1, while KS is 0 .

\section{CC BASEd Best BeAm Prediction}

We consider mmWave system best beam prediction by exploiting the sub-6 GHz links in a network-centric manner. In the considered NSA mmWave system, each UE can establish connection to the network both over a mmWave and a sub-6 GHz BS. In 5GNR, up to 64 beams are allowed to be utilized in mmWave frequencies. Thus, considerable overhead is incurred by the network for finding the best beam. Moreover, as sub-6 GHz channels are less vulnerable to blocking than mmWave channels, the long-term CSI of sub- $6 \mathrm{GHz}$ links is more reliable than of mmWave links.

\section{A. Beam Prediction Framework}

For each sub-6 $\mathrm{GHz}$ BS, a $\mathrm{CC}$ can be constructed and information of the mmWave system best beam can be added to the $\mathrm{CC}$ in an offline training phase. All information is gathered at a central control unit. Thus in the offline phase, the $\mathrm{CC}$ locations are annotated with best mmWave BS beam IDs. A prediction algorithm then predicts the best beam ID of mmWave system for the CC location of a new UE.
The considered network-centric best beam prediction model is illustrated in Fig. 1. The offline (training) phase is divided into three parts: CC construction, BS best beam ID annotation, and training of beam ID predictor. In the online phase, the UE is first mapped to a CC location, and based on the CC location, the best $\mathrm{BS}$ beam ID is predicted using the prediction algorithm,

The $\mathrm{CC}$ of sub-6 $\mathrm{GHz}$ BS $\mathcal{C}$ is constructed from the covariance matrices $\left\{\mathbf{R}_{u}\right\}_{u=1}^{U}$ of the UEs, in the offline phase. For each UE $u$, the best BS beam ID $m_{u}^{*}$ is attached to CC location $\mathbf{z}_{u}$. Then the prediction algorithm is trained based on the acquired data set. A supervised learning method learns the best beam ID mapping function $F\left(\mathbf{z}_{u}\right)$ which classifies the CC locations based on the best beam ID.

During the online phase, UE $i$ is communicating with the sub-6 GHz BS, which predicts its best beam ID using the trained beam ID predictor. First, the covariance matrix $\mathbf{R}_{i}$ of $\mathrm{UE} i$ at sub-6 $\mathrm{GHz}$ BS is estimated. An out-of-sample $\mathrm{CC}$ extension algorithm is applied to map the new UE on to the CC with coordinates $\mathbf{z}_{i}$. The best BS beam ID is then predicted based on the CC location of the UE, and then the BS can establish connection via the predicted BS beam in the mmWave band.

\section{B. Best Beam Classifiers}

To create the classifier $F\left(\mathbf{z}_{u}\right)$, classifying $\mathrm{CC}$ locations based on best beam IDs, standard ML techniques can be used. Here, we shall use a Support Vector Machine (SVM) as well as Neural Networks (NN). The K-Nearest Neighbor (KNN) best beam prediction is also considered, as a low-complexity alternative which needs no training.

$\mathrm{KNN}$ is a simple ML algorithm, where the classification is based on finding the $K$ nearest neighbors of a new sample in the training data set, based on a distance function. The data point is classified by a plurality vote of its neighbors, and assigned to the class most common among its $K$ nearest neighbors. If $K=1$, the data point is simply assigned to the class of the nearest neighbor. 
SVM is a ML technique that classifies linearly separable data in a high dimensional space into two classes. SVM transforms input data into a higher dimensional space through a kernel function in order to maximize the margin between two classes. Here, classes in 2D CC are sought for. SVM is designed for binary classification, but it can be extended to multiclass classification by breaking down the problem into several binary classification problems.

For multiclass SVM we use the One-vs-One method where every pair of two classes is classified by a binary classifier [13]. In this method $M(M-1) / 2$ classifiers are trained to classify $M$ classes. SVM solves a quadratic optimization problem to classify a point between two classes. After all pairs of classes are classified by a distinct classifier, a voting strategy is used to determine the class. The class with the majority of votes is assigned to that input location. Given a dataset of $I$ samples with features $\mathbf{z}_{i}$, i.e. the CC location used as features, and classes $y_{i} \in\{-1,1\}$, the SVM classification rule is:

$$
\phi(\mathbf{z})=\sum_{i=1}^{I} \alpha_{i} y_{i} \kappa\left(\mathbf{z}, \mathbf{z}_{i}\right)+\alpha_{0},
$$

where $\kappa\left(\mathbf{z}, \mathbf{z}_{i}\right)$ is the kernel function. Different kernel functions, depending on the training data, can be used in SVM. We used the Gaussian kernel $\kappa\left(\mathbf{z}, \mathbf{z}_{i}\right)=\exp \left(\frac{\left\|\mathbf{z}-\mathbf{z}_{i}\right\|^{2}}{\sigma^{2}}\right)$, where $\sigma$ is an adjustable hyper parameter for controlling overfitting. The equivalent optimization problem is:

$$
\min _{\alpha_{0}, \boldsymbol{\alpha}} \sum_{i=1}^{I}\left[1-y_{i} \phi\left(\mathbf{z}_{i}\right)\right]_{+}+\boldsymbol{\alpha}^{T} \mathbf{K} \boldsymbol{\alpha},
$$

where $\mathbf{K}$ is a square matrix obtained by applying the kernel function to all pairs of training data samples, the parameter vector is $\boldsymbol{\alpha}=\left[\alpha_{1}, \ldots, \alpha_{I}\right]^{T}$, and the softmax function $[x]_{+}=$ $\max (0, x)$ is used.

A NN consists of an input layer, hidden layers, and an output layer. In the problem at hand, the input layer gets a 2D input feature-the CC locations-and passes it through a series of fully connected hidden layers. The links between nodes have their own weights and a nonlinear activation function, which enables the NN to capture the non-linearity between input-output pairs. The best beam ID is a Multiclass classification problem, thus the last layer is equipped with a softmax function. The softmax layer outputs a vector of probabilities $\mathbf{f}(\mathbf{z}) \in \mathbb{R}^{M}$ which represents the probability that $m$ for $m=1, \ldots, M$ becomes the best beam ID for CC location $\mathbf{z}$; each beam is considered a class. The labels are "one-hot" encoded vectors, there is one dimension for each class. Thus the dimension of the output layer is $M$, the total number of mmWave BS beams, and beam $m$ is represented by the unit vector $\mathbf{y}_{m}$ in dimension $m$.

In the training phase, the output is fed to a Cross Entropy (CE) loss function $L=\sum_{u=1}^{U} \mathbf{y}_{u}^{T} \log _{2} \mathbf{f}\left(\mathbf{z}_{u}\right)$ to minimize the difference between the desired output and the model output. The weights and biases of the NN are selected aiming to minimize the total loss. The scaled conjugate gradient method is used in the back propagation to update model parameters.
TABLE I: Simulation Parameters

\begin{tabular}{l|cc}
\hline Parameter & sub-6 GHz & mmWave \\
\hline Center Freq. & $2 \mathrm{GHz}$ & $28 \mathrm{GHz}$ \\
Subcarrier BW. & $15 \mathrm{KHz}$ & $240 \mathrm{KHz}$ \\
No. of Subcarriers & 600 & 256 \\
BS antenna & $8 \mathrm{ULA}$ & $32 \mathrm{ULA}$ \\
BS antenna element pattern & Omnidirectional & 3GPP TR 36.873 \\
UE antenna & Omnidirectional & 8 ULA \\
Scenario & 3GPP 38.901 UMa-NLOS \\
\hline
\end{tabular}

Validation based early stopping is used to avoid overfitting. If after six consecutive epochs no improvement in validation loss is observed, we return to the best recorded model parameters.

The performance of a prediction scheme is evaluated in terms of average prediction accuracy. In addition, we shall measure top- $k$ prediction accuracy, i.e., whether the predicted beam is among the $k$ best ground truth beams.

\section{Simulations}

\section{A. Simulation Settings}

We model best beam prediction in a cellular system with a $2 \mathrm{GHz}$ and a $28 \mathrm{GHz}$ carrier. CSI for the communication links are generated using Quasi Deterministic Radio Channel Generator (QuaDRiGa) simulator [14]. The simulation settings are summarized in Table I.

The BS in the umbrella $2 \mathrm{GHz}$ network is located at $[-100,-100] \mathrm{m}$, the mmWave BS of interest is located at $[5,30] \mathrm{m}$ and UEs are sampled uniformly in a street segment between $\left[\begin{array}{ll}0 & 10\end{array}\right] \mathrm{m}$ on both the $\mathrm{x}$-axis and the y-axis. In the training phase, information is gathered from $3000 \mathrm{UE}$ locations scattered uniformly in the street segment. For each UE location we collect 100 small-scale fading samples for the channel covariance estimation at the $2 \mathrm{GHz} \mathrm{BS}$.

For each sample UE location, the best mmWave beam is found by a beam management protocol run between the UE and the mmWave BS. The BS transmits with different beams, the UE selects the instantaneously best beam for each BS beam, averages the received power across frequency and temporal samples, and feeds back best beam information to the BS. We collect 100 temporal samples of the mmWave channel for each location for annotating CC locations with the corresponding best mmWave BS beam. The temporal samples are collected over a $100 \mathrm{~ms}$ period, assuming that UEs move on a straight line with a $50 \mathrm{~km} / \mathrm{h}$ speed. In Fig. 2, the $10 \times 10$ $\mathrm{m}$ street segment is depicted, with 3000 ground truth sample locations of the UEs, and a color coding according to the best mmWave beam.

\section{B. Annotated CC}

The computed covariances of the $2 \mathrm{GHz}$ channels are used to calculate dissimilarities, and a $\mathrm{CC}$ is constructed from the dissimilarity matrix using the ISOMAP (with 12 neighbors) and $t$-SNE (with perplexity value of 100) DR techniques. Fig. 3 shows the resulting annotated $\mathrm{CCs}$ obtained with ISOMAP and $t$-SNE dimensionality reduction. 


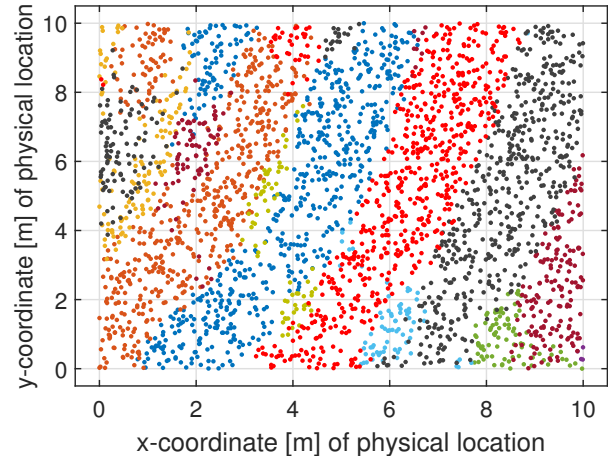

Fig. 2: The true physical locations marked with their best BS beams with different colors. For example, beam 32 is the best beam for the locations marked with red.
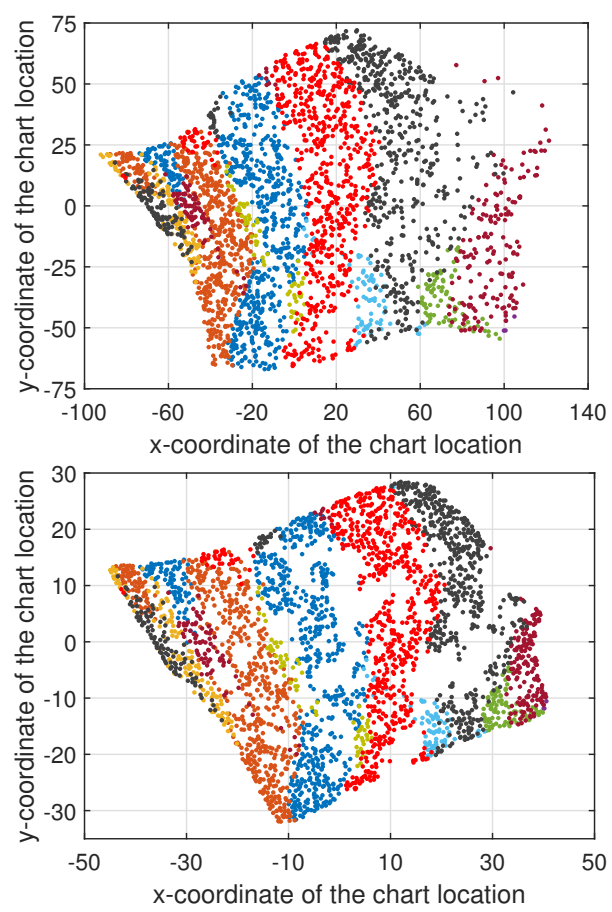

Fig. 3: CC annotated with best beam information using: (Top); ISOMAP DR. (Bottom); $t$-SNE DR.

The CCs show good approximation of the physical locations of the UEs with the general global structure and spatial distribution pattern maintained by the $t$-SNE CC in particular. The quality metrics CT, TW and KS are tabulated in Table II. They show that the CCs have good quality. The values of CT and TW of over 0.99 indicate that the CCs preserve the neighborhood relations between the UEs in the physical locations well. The KS values confirm the visual perception that $t$-SNE preserves the global geometry better than ISOMAP.

TABLE II: CC Performance Measures for 50 Nearest Neighbors

\begin{tabular}{l|ccc}
\hline DR & CT & TW & KS \\
\hline ISOMAP & 0.999 & 0.999 & 0.184 \\
$t$-SNE & 0.998 & 0.998 & 0.148 \\
\hline
\end{tabular}

\section{Best Beam ID Prediction}

The best beam prediction accuracy is evaluated for KNN, SVM and NN predictors. For KNN, we consider two cases: the nearest neighbor predictor $\mathrm{KNN}(1)$, and $\mathrm{KNN}(10)$ based on voting by the 10 nearest neighbors. The Euclidean distance in the chart is used to determine the neighbors. When creating an SVM based beam prediction function, a Gaussian kernel is used. For NN based prediction, the hyperbolic tangent activation function is used for the hidden layers. Three hidden layers was found to provide best performance.

We consider two benchmarks for CC-based prediction; best beam ID prediction based on the true physical location, and based on Covariance Fingerprinting (Cov-FP). The Cov-FP feature is obtained by stacking the elements of the sub- 6 $\mathrm{GHz}$ covariance matrix as a vector of real values. Regression learners are constructed based on both of these cases. Note that in the literature on NSA beam prediction [5], it is assumed that the user, using GPS or other techniques, determines its location, and transmits it using the sub- $6 \mathrm{GHz}$ control channel. The best BS beam and UE beam are then determined at the UE side and transmitted using sub- $6 \mathrm{GHz}$ control channel in the training phase. This operation needs UE involvement in the process. In our approach, there is no need for location information to predict the best beam ID.

For prediction performance evaluation, we divide the entire data set for all algorithms into training and test sets. The training set has 70\% (2100) of the UEs from the dataset selected randomly. The test set is comprised of the remaining 30\% (900) of the UEs. The prediction accuracy of all algorithms is averaged over 100 random divisions to training and test sets.

The best beam prediction accuracy of different NNs for various inputs is shown in Fig. 4. Both the accuracy in predicting the best beam (Top-1) and in finding one of the two best beams (Top-2) is depicted. For true location, the best NN structure has 20 nodes per layer, while 30 nodes is best for ISOMAP as well as covariance FP, and 50 nodes for $t$-SNE. By adding more hidden layers and neurons no improvement is gained, and the structure gets more complicated.

The average prediction accuracies for CC-based and benchmark predictors using KNN, SVM and NNs are compared in Table III. For all inputs, NN outperforms SVM, which

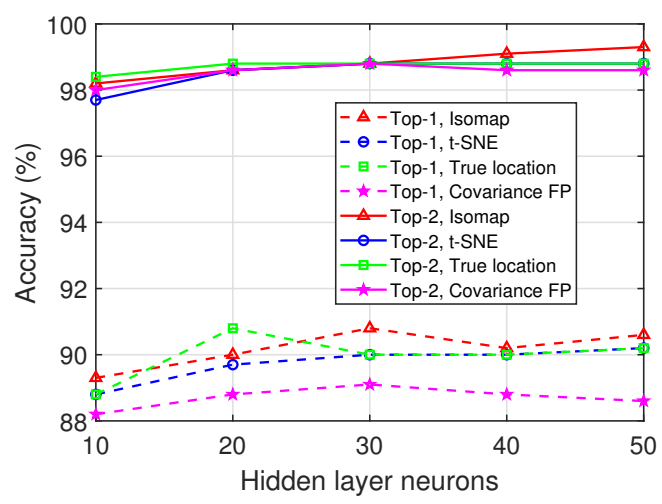

Fig. 4: NN with different number of hidden layer neurons, top-1 and top-2 accuracy. 
TABLE III: Prediction Accuracy of Different Predictors

\begin{tabular}{l|cccc}
\hline Predictor & Best NN & SVM & KNN(10) & KNN(1) \\
\hline ISOMAP & 90.8 & 88.4 & 88.0 & 88.2 \\
$t$-SNE & 90.2 & 88.2 & 86.6 & 86.8 \\
True location & 90.8 & 89.3 & 88.4 & 87.1 \\
Cov-FP & 89.1 & 87.7 & 85.5 & 86.0 \\
\hline
\end{tabular}

again outperforms KNN. The accuracy of the best predictor (i.e., NN) based on ISOMAP CC achieves the accuracy of prediction based on true location. The CC-based algorithms outperform direct long-term CSI-fingerprinting, and the margin increases when predictor complexity decreases. The performance benefit from using $\mathrm{CC}$ for beam prediction is due to the dimensionality reduction extracting information of the whole CSI sample set, merging it to a proximity encoded in the $\mathrm{CC}$, while fingerprinting is based only on pairwise CSI dissimilarities.

In Table IV, the prediction accuracy of top- 1 , top- 2 and top-3 best mmWave beams for best NN based predictors are summarized. The top- 2 or top-3 information can be used to reduce the best beam search time, if this information is shared with the UEs. Accordingly, instead of searching over 32 beams, the search is carried over 2-3 beams.

To assess the complexity of CC-based prediction, the online phase is of particular interest as it can increase the overhead. For CC-based algorithms, the dominant computational complexity lies in finding the out-of-sample CC-location, while for Cov-FP, the complexity arises from the high dimensionality of predictor input. If out-of-sample extension is done in straight forward manner, its complexity is comparable to using Cov-FP complexity arising from using covariance directly as input to the predictors. It is possible to reduce out-of-sample complexity for $\mathrm{CC}$, as well as the KNN complexity for CovFP, using a hierarchical approach. Accordingly Cov-FP based on NN and SVM remain the most complex solutions.

\section{CONClusion}

In this paper, we have considered a best BS beam prediction scheme for NSA mmWave systems based on channel charting. The prediction is based on channel chart which is a radio map of a sub-6 $\mathrm{GHz}$ cell based on the received signals at a BS, which is annotated with information of the best mmWave beams. Beam prediction is based on a networkcentric approach where all the processing is performed in the network. The prediction framework involves an offline training phase and an online prediction phase. During the training phase, the CC is constructed at sub-6 GHz BS. The best BS beam for UEs in the CC at the mmWave BS is determined based on the UE transmissions towards BS beams. A support

TABLE IV: Top-1, Top-2 and Top-3 Prediction Accuracy of Best NN Prediction Schemes

\begin{tabular}{l|ccc}
\hline Input & Top-1 & Top-2 & Top-3 \\
\hline True location & 90.8 & 98.8 & 99.7 \\
$t$-SNE & 90.2 & 98.8 & 99.7 \\
I SOMAP & 90.8 & 99.3 & 99.5 \\
Cov-FP & 89.1 & 98.8 & 99.7 \\
\hline
\end{tabular}

vector machine and neural networks are used for creating the beam prediction function. In addition, K-nearest neighbor prediction is considered as a benchmark for its simplicity. We performed simulations with spatially consistent channels generated by the QuaDRiGa simulator, and evaluated the prediction accuracies of the proposed framework based on KNN, SVM and NN. The performance of NN based prediction outperforms KNN and SVM. The best BS beam prediction based on CC using NN matches the performance of a predictor based on true location, with $90.8 \%$ accuracy. The presented results show that $\mathrm{CC}$ can be used as a replacement for true location based RRM.

\section{ACKNOWLEDGMENT}

This work was funded in part by the Academy of Finland (grant 319484) and the European Union under the framework of the project H2020-MSCA-ITN 813999 Windmill.

\section{REFERENCES}

[1] S. Rangan, T. S. Rappaport, and E. Erkip, "Millimeter-wave cellular wireless networks: Potentials and challenges," Proc. of the IEEE, vol. 102, no. 3, pp. 366-385, Mar. 2014.

[2] M. Giordani, M. Polese, A. Roy, D. Castor, and M. Zorzi, "Standalone and non-standalone beam management for 3GPP NR at mmWaves," IEEE Commun. Magazine, vol. 57, no. 4, pp. 123-129, Apr. 2019.

[3] M. Giordani, M. Mezzavilla, and M. Zorzi, "Initial Access in 5G mmWave Cellular Networks," IEEE Commun. Magazine, vol. 54, no. 11 , pp. 40-47, Nov. 2016.

[4] V. Va, T. Shimizu, G. Bansal, and R. W. Heath, "Position-aided millimeter wave V2I beam alignment: A learning-to-rank approach," in IEEE PIMRC, Oct. 2017, pp. 1-5.

[5] M. Arvinte, M. Tavares, and D. Samardzija, "Beam management in 5G NR using geolocation side information," in Proc. of Conf. on Information Sciences and Systems (CISS), Mar. 2019, pp. 1-6.

[6] A. Pan, T. Zhang, and X. Han, "Location information aided beam allocation algorithm in mmWave massive MIMO systems," in Proc. of IEEE Internat. Conf. on Commun. in China (ICCC), Oct. 2017, pp. $1-6$.

[7] S. Tomasin, C. Mazzucco, D. De Donno, and F. Cappellaro, "Beamsweeping design based on nearest users position and beam in $5 \mathrm{G}$ mmWave networks," IEEE Access, vol. 8, pp. 124402-124413, 2020.

[8] M. Alrabeiah and A. Alkhateeb, "Deep learning for mmWave beam and blockage prediction using Sub-6 GHz channels," IEEE Trans. on Commun., vol. 68, no. 9, pp. 5504-5518, Sept. 2020.

[9] C. Studer, S. Medjkouh, E. Gönültaş, T. Goldstein, and O. Tirkkonen, "Channel charting: Locating users within the radio environment using channel state information," IEEE Access, vol. 6, pp. 47682-47698, 2018.

[10] S. Bonnabel and R. Sepulchre, "Riemannian metric and geometric mean for positive semidefinite matrices of fixed rank," SIAM Journal on Matrix Analysis and Applications, vol. 31, no. 3, pp. 1055-1070, July 2009.

[11] L. van der Maaten, E. Postma, and H. Herik, "Dimensionality reduction: A comparative review," JMLR, vol. 10, Jan. 2007.

[12] J. Deng, S. Medjkouh, N. Malm, O. Tirkkonen, and C. Studer, "Multipoint channel charting for wireless networks," in Proc. of Asilomar Conf. on Signals, Systems, and Computers, Oct. 2018, pp. 286-290.

[13] R. Soentpiet et al., Advances in kernel methods: support vector learning. MIT press, 1999

[14] S. Jaeckel, L. Raschkowski, K. Borner, and L. Thiele, "QuaDRiGa: A 3-D multi-cell channel model with time evolution for enabling virtual field trials," IEEE Trans. Antennas Propag., vol. 62, no. 6, pp. 32423256, Jun. 2014. 